\title{
Téoros
}

Revue de recherche en tourisme

\section{Tourisme et autochtones}

\section{Yves Archambault}

Volume 29, numéro 1, 2010

Tourisme et autochtones

URI : https://id.erudit.org/iderudit/1024756ar

DOI : https://doi.org/10.7202/1024756ar

Aller au sommaire du numéro

\section{Éditeur(s)}

Université du Québec à Montréal

\section{ISSN}

0712-8657 (imprimé)

1923-2705 (numérique)

Découvrir la revue

\section{Citer ce document}

Archambault, Y. (2010). Tourisme et autochtones. Téoros, 29(1), 67-68. https://doi.org/10.7202/1024756ar d'utilisation que vous pouvez consulter en ligne.

https://apropos.erudit.org/fr/usagers/politique-dutilisation/ 
NDLR : Dans la continuité des changements mis en place depuis 3 numéros, Téoros poursuit sa transformation pour compléter, avec cette édition, sa nouvelle forme. Désormais, Téoros ajoute un second dossier thématique à sa présentation. Ce dossier additionnel vous permettra ainsi d'étudier des thématiques plus pointues ou de revoir des sujets déjà traités par le passé et qui ont évolué. Les articles présentés dans ce second dossier suivent les mêmes principes de coordination que notre dossier principal, parrainé par un rédacteur invité et soumis au processus de révision par les pairs.

Pour cette édition, le second dossier est consacré au tourisme et aux autochtones. La rédaction de Téoros profite de cette occasion pour vous inviter à soumettre vos propositions de textes ou de dossiers thématiques.

\title{
Tourisme et autochtones
}

\author{
Yves ARCHAMBAULT \\ Agent de recherche et de planification socio-économique \\ Ministère de l'Immigration et des Communautés culturelles du Québec \\ Yves.Archambault@micc.gouv.qc.ca
}

On assiste depuis quelques décennies à un élargissement des horizons touristiques. Traditionnellement confiné aux destinations classiques de notoriété internationale, le tourisme mondial s'est considérablement diversifié au cours des années et cible notamment les collectivités autochtones. Relativement nouvelle, cette vocation de l'industrie touristique répond à une certaine quête de la nouveauté, de l'authenticité, voire de l'inédit, chez les voyageurs, d'autant plus qu'elle coïncide avec l'émancipation des peuples autochtones et la reconnaissance de leur statut et de leurs cultures à travers le monde. Aussi, assiste-t-on à la prolifération des infrastructures d'accueil des touristes dans des aires de peuplement autochtone autrefois isolées et peu accessibles aux étrangers : aéroports, hôtels et autres structures d'hébergement, restauration adaptée aux goûts des touristes, agences locales spécialisées dans la découverte de communautés indigènes et des modes de vie traditionnels.

L'expérience touristique en milieu indigène peut être aussi bien le fait de grandes organisations touristiques internationales, axées sur le mode des voyagistes de masse, que de petits groupes de visiteurs curieux de connaitre davantage les cultures autochtones. Elle peut également être caractérisée par la prise en charge, partielle ou entière, des activités touristiques par ces communautés elles-mêmes. Ainsi, on observe à travers le monde divers modes de gestion des activités touristiques par les communautés indigènes locales, qu'il s'agisse de l'autogestion complète ou d'une forme ou une autre de cogestion avec les représentants de l'industrie du tourisme mondial. Il peut s'agir de tourisme mercantile aussi bien que de tourisme solidaire.

Dans quelle mesure l'afflux plus ou moins massif de visiteurs étrangers en milieu autochtone perturbe-t-il le mode de vie de ces communautés soudées par un système de valeurs traditionnelles? Quels sont les impacts du tourisme moderne sur ces populations hôtes aux plans économique, socioculturel et des pratiques linguistiques, et sur l'environnement physique? Assiste-t-on à une mercantilisation des activités traditionnelles liées à la survie de ces communautés, à une déstructuration des tissus familial et communautaire, à une déperdition accélérée de la langue vernaculaire locale, ou à la dégradation de l'environnement naturel régional? Voilà autant de questionnements qui ont jusqu'à présent guidé la réflexion sur le tourisme autochtone.

En 1998, Téoros avait consacré un numéro à ces questions, offrant un recueil de témoignages de divers acteurs du secteur du tourisme en milieu autochtone québécois. On 
y examinait les impacts socioéconomiques du tourisme au sein des communautés amérindiennes du Québec, sur un mode essentiellement descriptif. On y avait alors bien décrit les divers problèmes auxquels était confrontée l'industrie du tourisme autochtone et ses acteurs.

Plus d'une décennie plus tard, Téoros explore à nouveau cette thématique, cette fois dans le but de s'attarder aux solutions apportées, ici et ailleurs, aux enjeux problématiques du tourisme autochtone.

Ce dossier traite des enjeux relatifs à la prise en charge par des communautés autochtones de l'activité touristique dans diverses régions du monde. Ainsi, Sylvie Blangy, Raynald Harvey Lemelin et Robin McGinley se penchent sur la mise sur pied d'une offre de séjour touristique respectant les valeurs des populations hôtes au sein des communautés cris du Canada, et ce, sur la base de la recherche-action participative faisant appel à la mobilisation et au partage des connaissances et responsabilités entre Européens et autochtones.

Pour sa part, Jean-Philippe Principaud fait état d'une expérience de projet touristique dans un contexte d'instabilité politique : il s'intéresse à la difficile relance d'un projet de tourisme rural intégré en Basse-Casamance au Sénégal interrompu à la suite d'une rébellion.
Enfin, Aurélie Condevaux examine la question des conflits et des malentendus engendrés par la mise en présence de systèmes de valeurs et de normes différents lors de manifestations culturelles polynésiennes auprès de touristes occidentaux. Son texte nous rappelle que, même si des communautés s'impliquent aujourd'hui dans la gestion du tourisme, des difficultés de communication persistent.

Dans le prolongement de ce numéro, d'autres aspects de la question du tourisme dans les communautés autochtones auraient pu être explorés ou approfondis, tels que les mutations socioculturelles des populations hôtes, l'examen des différents modes de gestion partagée des lieux touristiques, la gestion particulière des sites sacrés, ou encore l'impact de l'afflux touristique sur l'environnement physique du milieu autochtone. Cependant, les études présentées ici soulignent combien la relation entre le phénomène touristique et les communautés autochtones a évolué ces dernières années. Il est fort probable que de nombreux chercheurs continueront à travailler sur ces questions pour comprendre les enjeux liés à la gestion et à l'aménagement de l'activité touristique en milieu indigène, mais aussi les répercussions culturelles et socioéconomiques sur le mode de vie et les valeurs des sociétés autochtones, dorénavant impliquées dans la gestion du tourisme.

Bonne lecture!

\section{Appel à textes}

En dehors des dossiers thématiques, Téoros ouvre ses pages aux propositions spontanées. La rédaction invite tous les chercheurs qui œuvrent dans le domaine du tourisme ou qui s'intéressent au tourisme sous tous ses aspects à soumettre des articles de nature analytique à la revue. On peut soumettre un article en l'envoyant à :

\section{teoros@uqam.ca}

Les textes soumis doivent apporter une contribution scientifique originale, que ce soit par le biais d'information factuelle jusqu'alors inconnue ou par une nouvelle interprétation d'un thème particulier. Téoros vise avant tout le transfert de connaissances; son objectif est donc de promouvoir une meilleure compréhension des phénomènes liés au tourisme.

Les auteurs doivent faire parvenir un manuscrit présenté selon les règles de la revue, disponibles au www.teoros.uqam.ca. Un article analytique compte environ 6000 mots et n'excède pas 8000 mots, avec trois ou quatre illustrations en 300 ppp.
Les articles peuvent être soumis en anglais ou en français et doivent être accompagnés d'un résumé de 200 mots et de cinq mots-clés.

La publication des articles se fait sous réserve d'une évaluation. Tous les manuscrits seront évalués anonymement par des pairs, qui pourront faire des suggestions ou demander des modifications. La rédaction transmettra l'avis des évaluateurs aux auteurs et s'assurera que les modifications demandées seront apportées.

Au plaisir de vous lire dans nos pages.

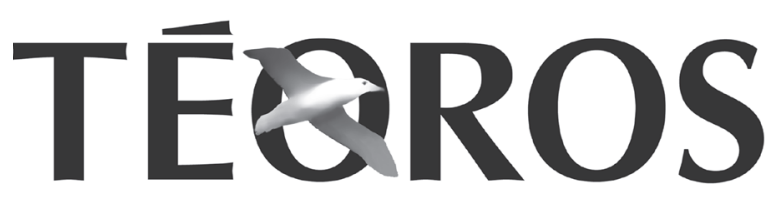

REVUE DE RECHERCHE EN TOURISME 